\title{
Effect of Modified Clamshell Exercise on Gluteus Medius, Quadratus Lumborum and Anterior Hip Flexor in Participants with Gluteus Medius Weakness
}

\author{
Seom-Gyeul Jeong $\cdot$ Heon-Seock Cynn ${ }^{\dagger} \cdot$ Ji-Hyun Lee $\cdot$ Silah Choi $\cdot$ Daeun Kim
}

Department of Physical Therapy, Graduate School, Yonsei University

Received: November 21, 2018 / Revised: January 9, 2019 / Accepted: January 31, 2019

(c) 2019 J Korean Soc Phys Med

\section{| Abstract |}

PURPOSE: This study compared the effects of three different clamshell exercises (CLAM) on the gluteus medius (GMED), quadratus lumborum (QL), anterior hip flexor (AHF), gluteus medius/quadratus lumborum ratio, and gluteus medius/anterior hip flexor ratio by studying the activities of participants with GMED weakness.

METHODS: Eleven subjects with weak GMED participated in this study. Subjects performed CLAM under three different conditions (standard, and modified 1 and 2). Surface electromyography was then used to measure the muscle activity and one-way repeated-measures analysis of variance was used to assess the statistical significance of the measured variables.

RESULTS: GMED and the QL muscle activities did not differ significantly between the standard CLAM and the modified CLAM with the 2 different foot positions $(\mathrm{F}=4.74$, $\mathrm{P}=.02 ; \mathrm{F}=4.57, \mathrm{P}=.02$, respectively). $\mathrm{AHF}$ activity was

$\dagger$ Corresponding Author : Heon-Seock Cynn cynn@yonsei.ac.kr, http://orcid.org/0000-0002-5810-2371

This is an Open Access article distributed under the terms of the Creative Commons Attribution Non-Commercial License (http://creativecommons.org/licenses/by-nc/3.0) which permits unrestricted non-commercial use, distribution, and reproduction in any medium, provided the original work is properly cited. significantly different in the two different foot positions when compared to the standard CLAM $(\mathrm{F}=11.17, \mathrm{P}=.00)$. However, there was no significant difference between the AHF activities for the two different foot positions $(\mathrm{P}=.09)$. Finally, GMED/QL and GMED/AHF ratios were not significantly different between the three different CLAM exercises $(\mathrm{F}=.63$, $\mathrm{P}=.55 ; \mathrm{F}=.82, \mathrm{P}=.45$, respectively).

CONCLUSION: Modified CLAM can be recommended as a good method to minimize AHF activity while maintaining GMED activity in subjects with weak GMED.

Key Words: Anterior hip flexor, Clamshell exercise, Electromyography

\section{Introduction}

The gluteus medius (GMED) has a crucial kinesiology role in human gait and load migration through the hip joint [1]. There are two major functions of the GMED during hip movement. First, Gottschalk et al. [2] defined that the GMED is a hip stabilizer stabilizing the femoral head in the acetabulum during different hip rotations. Second, functions of the GMED include initiation of hip abduction (open kinematic chain) and lateral pelvic tilting (closed kinematic chain). In other words, individuals with hip abductor weakness have GMED weakness, which leads to compensatory motion of the lower back, hip, and knee [3-5]. 
For example, in terms of the hip and knee, a contemporary clinical theory that may explain the cause of patellofemoral pain syndrome and iliotibial band syndrome is that proximal hip muscle weakness leads to dynamic valgus of the knee joint [6]. Ankle hyper-pronation, genu valgum, tibial internal rotation, femoral adduction and internal rotation occur when the hip muscles cannot get over the external torque caused by gravity acting on the body's center of mass [6]. Moreover, in terms of the lower back, lateral tilt of pelvic can cause when the quadratus lumborum (QL) substitutes for a weakened GMED [7]. Substitution by the lateral portion of the QL leads to lateral instability and impaired movement caused by lumbar spine lateral flexion and pelvic obliquity (lateral pelvic tilt) [8]. In addition, increased tension in the QL is implicated in pelvic upward movement and rotational mal-alignment [9]. Clinicians often report over-activity and trigger points for the QL with GMED insufficiency in patients with back pain [7]. In patients with low back pain, GMED activity is delayed, whereas GMED activity has been observed before the ipsilateral QL in normal participants. Recruitment imbalance between the GMED and QL can induce movement impairment [5].

Previous studies have suggested a treatment protocol that included exercise to facilitate the recruitment of the GMED and relaxation to reduce the activity of the QL $[7,10]$. Other studies demonstrated that QL muscle activity could be decreased by using a device to provide pressure biofeedback or a pelvic compression belt during side-lying hip abduction [4,11]. In the clinical setting, two of the most common GMED strengthening exercises are side-lying clamshell (CLAM) and side-lying hip abduction exercises [12]. Previous studies have reported that the GMED-to-tensor fasciae latae (TFL) peak EMG signal amplitude ratio was far greater for the standard CLAM than that for side-lying abduction exercise, and suggested that the standard CLAM may be the preferred rehabilitative exercise when minimal TFL muscle activation is desired
$[13,14]$. CLAM activity incorporates open-chain hip external rotation and abduction, and is often used very early in rehabilitation when weakness of the abductors and external rotators exists [15]. However, previous studies have reported that the CLAM activates the anterior hip flexor (AHF) to a greater extent, but does not produce high activation of the GMED, and high levels of AHF activity during CLAM are came from the need to keep the hip in a flexed position while the hip external rotation movement is performed [15]. Moreover, for the CLAM with the hip flexion at 45 degrees, the AHF is more active than the GMED. However, the activities or the ratio between the GMED, QL, and AHF muscles during CLAM exercises have not been examined in previous studies.

During CLAM exercises, along with GMED activation, it is important to minimize the QL and AHF activity. Thus, the principal investigator (PI) in the current study developed modified CLAM exercises to reduce AHF activity via a mechanism of reciprocal inhibition. Briefly, subjects were instructed to push their feet against a wall at about 10\% of the maximal contraction while they were performing the CLAM [16,17]. The purpose of this study was to compare the GMED, QL, and AHF muscle activities and the GMED/QL and GMED/AHF muscle activity ratios between the standard CLAM and modified CLAM 1 and 2 (with two different foot positions) among participants with gluteus medius weakness. We hypothesized that the GMED, QL, and AHF activities would be different during both modified CLAM 1 and 2 in contrast to during the standard CLAM among these participants.

\section{Methods}

\section{Participants}

G-power software 3.1 (Franz Faul, University of Kiel, Kiel, Germany) was used for power analysis. The required sample size of the 10 participants was calculated from the data collected based on a pilot study of three participants 
Table 1. Descriptive Characteristics of the Study Population

\begin{tabular}{cccc}
\hline Variables & Male $(\mathrm{n}=7)$ & Female $(\mathrm{n}=4)$ & Total $(\mathrm{n}=11)$ \\
\hline Age $($ years $)$ & $22.714 \pm 1.889$ & $21.250 \pm .500$ & $22.181 \pm 1.662$ \\
Height $(\mathrm{cm})$ & $175.428 \pm 3.457$ & $162.500 \pm 3.316$ & $170.727 \pm 7.281$ \\
Weight $(\mathrm{kg})$ & $68.614 \pm 4.906$ & $56.925 \pm 3.441$ & $64.363 \pm 7.264$ \\
BMI $\left(\mathrm{kg} / \mathrm{m}^{2}\right)$ & $22.285 \pm 1.319$ & $21.575 \pm .850$ & $22.027 \pm 1.179$ \\
\hline
\end{tabular}

Abbreviations: BMI; body mass index. Values are presented as the means $\pm \mathrm{SD}$.

to obtain a power of .80 and an effect size of .41 (calculated by partial $\eta 2$ of .148 from the pilot study) with an a level of .05. Eleven participants ( 7 males, 4 females) with weak GMED that were selected through manual muscle testing participated in this study. Table 1 indicates the demographic information of the participants.

The inclusion criteria included the subjects being able to sustain 5 seconds of isometric hip abduction in the side-lying position [18]. To confirm the GMED weakness, the participants assumed a side-lying position on the treatment table. Each participant's bottom leg was flexed for stability and comfort, and the test leg was parallel to the rest of the trunk. The investigator's hand was placed at $10 \mathrm{~cm}$ proximal to the lateral femoral epicondyle and the hip of the test limb was abducted to half of the hip abduction total ROM. [19]. The principal investigator (JSG) provided verbal encouragement to facilitate the maximal performance and gave instructions to prevent hip medial rotation or flexion through recruitment of the TFL or any pelvic hiking through recruitment of the QL [20]. The participants took a 3-minute rest between the 2 trials [21]. Muscle strength was graded based on the method represented by Kendall et al. [22] as 0, 1, 2, 3, 4, or 5 out of 5, then grouped as either 'strong' (4 or 5/5) or 'weak' (3/5 or less) [23]. The reliability for individual muscle groups ranged from .63 to .93 [22].

This study excluded the participants with past or present musculoskeletal, neurological, or cardiopulmonary diseases, shortness of iliotibial band and calf muscles, and limited ROM of the hip, knee, and ankle joints that could interfere with side-lying position. Overweight or obese participants were also excluded, as fatty tissue could interfere with EMG signals, acting as a low-pass filter [24]. The participants were identified as 'overweight' and 'obese,' if they presented with a body mass index (BMI) $>25$ [25]. BMI is defined as a participant's weight divided by the square of his or her height, in units of $\mathrm{kg} / \mathrm{m}^{2}$. All participants signed a written consent form authorized by the Yonsei University Wonju Institutional Review Board for Human Investigations, prior to the participation in the study.

\section{Surface EMG recording and data processing}

Surface EMG data were collected by using a Tele-Myo DTS EMG instrument with a wireless telemetry system (Noraxon, Inc., Scottsdale, AZ, USA) at a sampling rate of $1000 \mathrm{~Hz}$. A digital band-pass filter (Lancosh FIR), which filtered the raw signals, was between 20 and $450 \mathrm{~Hz}$, and a common mode rejection ratio of $92 \mathrm{~dB}$ at $60 \mathrm{~Hz}$ was obtained. Myo-Research Master Edition 1.06 XP software analyzed the EMG data and root-mean-square values were calculated with a moving window of $50 \mathrm{~ms}$. The data were collected from the GMED, QL, and AHF muscles on the side of the participant with the GMED weakness. An investigator prepared the electrode sites by cleaning the skin with isopropyl alcohol using a sterile gauze pad and shaving the participant's hair from the immediate vicinity of the muscle bellies to diminish impedance to the EMG signal, after which disposable $\mathrm{Ag} / \mathrm{AgCl}$ surface electrodes were fixed on the proper sites [26,27]. Electrodes were positioned over the midsection of the participant's muscle 
Table 2. Electromyographic Activities and Activity Ratios During Exercises

\begin{tabular}{cccccc}
\hline & \multicolumn{3}{c}{ Exercise } & \multirow{2}{*}{ F } & p \\
\cline { 2 - 4 } & Standard CLAM & Modified CLAM 1 & Modified CLAM 2 & & \\
\hline Gmed & $32.887 \pm 16.399$ & $20.255 \pm 13.073$ & $24.171 \pm 11.259$ & 4.747 & .034 \\
QL & $25.263 \pm 17.163$ & $19.065 \pm 12.813$ & $19.798 \pm 12.456$ & 4.576 & .027 \\
AHF & $30.852 \pm 22.044$ & $12.917 \pm 13.798$ & $17.171 \pm 12.269$ & 11.173 & $.003^{*}$ \\
Gmed/QL & $3.065 \pm 3.889$ & $2.161 \pm 2.547$ & $2.221 \pm 2.168$ & .869 & .391 \\
Gmed/AFH & $2.199 \pm 3.271$ & $3.444 \pm 4.159$ & $2.480 \pm 2.524$ & .824 & .425 \\
\hline
\end{tabular}

Values are presented as the mean \pm SD.

Gmed: gluteus medius.

QL: quadratus lumborum.

AHF: anterior hip flexor.

${ }^{*} \mathrm{p}<.017$, by one way repeated analyses of variance.

bellies parallel to the target muscle fibers. Two electrodes were placed about $20 \mathrm{~mm}$ apart in the direction of the muscle fibers. For the GMED muscle, the electrodes were placed one-third of the distance between the greater trochanter of the femur and the iliac crest, directly above the greater trochanter of the femur. For the AHF muscle, the electrodes were placed medial to the palpable mass of the quadriceps femoris and in the femoral triangle just lateral to the femoral pulse below the inguinal ligament. For the QL muscle, the electrodes were placed at a slightly oblique angle at half the distance between $4 \mathrm{~cm}$ lateral from the vertebral ridge to the belly of the erector spinae muscle and the 12th rib and the iliac crest. Appropriate placement of the electrodes was confirmed by observing the participants while they completed five repetitions of side-lying hip abduction. Electrode contacts were checked before all contractions [26].

The maximum voluntary isometric contractions (MVICs) in the standard manual muscle-test position were used to normalize the GMED, QL, and AHF [22]. EMG data were collected for 5 seconds during the isometric phase, and results were calculated from the middle 3 seconds of each exercise to avoid any possible starting or ending effects and connecting element of the skinelectrode [28,29]. To obtain the MVIC values for the
GMED, the participants assumed a side-lying position on the treatment table with the knee flexed at $90^{\circ}$ for stability and the test leg up and the bottom hip flexed at $45^{\circ}$. The test hip was placed in extension and in slight lateral rotation and the leg was abducted to approximately $50 \%$ of the hip abduction. An investigator applied inferior force to the ankle while keeping the hip with the other hand. To obtain the MVIC values for the AHF, an investigator stabilized the opposite iliac crest with the participants assuming a supine position on the treatment table. The quadriceps of the test leg stabilized the knee in extension, after which the hip was flexed in a position of slight abduction and slight lateral rotation. An investigator applied force to the anteromedial aspect of the test leg in the direction of extension and slight abduction, directly opposite the line of pull of the psoas major from its origin from the lumbar spine to its insertion on the lesser trochanter of the femur. To collect the MVIC values for the QL, the participants assumed a prone position on the treatment table with the test leg placed in the degree of abduction that corresponds with the line of the fibers of the QL and in slight extension. An investigator applied traction to the test leg, directly opposing the line of the QL. The mean of the MVIC values estimated from the two trials for 5 seconds with a 3-minute rest between the contractions was used for MVIC data 


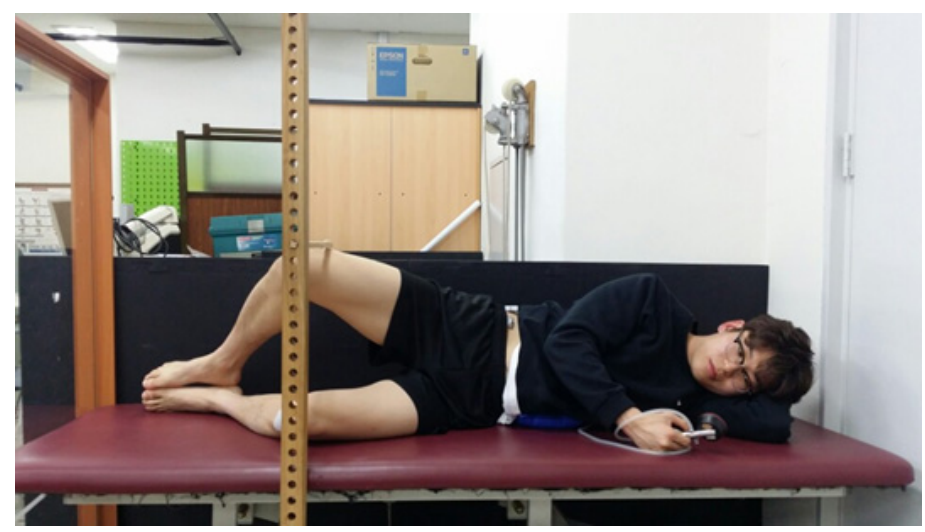

Fig. 1. Standard clamshell exercise (CLAM); both feet and back are not in contact with the wall

analysis. The participants had a 3-minute rest between muscle tests [29]. The EMG amplitude of each exercise was expressed as a percentage of the average MVIC value for each muscle ( $\%$ MVIC) because this has been reported to be the most credible method of EMG normalization for hip abduction exercises [3]. To calculate the muscle activity ratios of the GMED/AHF and GMED/QL, the normalized GMED amplitude was divided by the normalized AHF and QL amplitudes, respectively.

\section{Experimental Procedures}

The muscle activities of the GMED, QL, and AHF were determined during the three types of CLAM exercises (standard and modified with the two different foot positions). The participants were instructed to draw lots to randomize the exercise order to avoid learning effects or fatigue. Each participant performed all the exercises with the weak GMED side for both the lower extremities. A metronome was set at one beat per second to ensure that each participant performed the exercises at a standard speed. [30]. For all CLAMs, the participants performed three trials for each exercise condition with a 3-minute rest between exercises [31]. The mean value was used for data analysis [32]. To minimize any potential learning effect, all participants performed one practice set of each exercise before the data collection and were instructed by the principal investigator on how to perform the exercises $\{\mathrm{PI}$, JeongSeomGyeul (JSG)\}. During all the trials, the investigators gave oral feedback to correct the errors, as well as to assist in maintenance of the proper tempo.

\section{1) Standard CLAM}

To perform the standard CLAM, participants assumed a side-lying position with the weak limb up, both hips flexed at $45^{\circ}$, the knees flexed at $90^{\circ}$, and neither the feet nor back not in contact with the wall (Fig. 1). Keeping both their heels and the first metatarsal head together, the subjects separated their knees and rotated the weak limb upward. The target bar was positioned such that the top of the weak side's knee touched the bar when the angle between the top leg and the horizontal plane became $25^{\circ}$. The participants were instructed not to tip it backward and to hold the pelvis in a neutral position, and this was monitored visually and using a stabilizer pressure bio-feedback unit (Chattanooga Group, Inc, Hixson, TN, USA). This unit composed of an inflatable air bag linked to a pressure gauge. When it is placed below the trunk between the distal ribs and the iliac crest, changes in the body's position are reflected as variations in pressure, which the participant views. This provides additional feedback for unwanted 


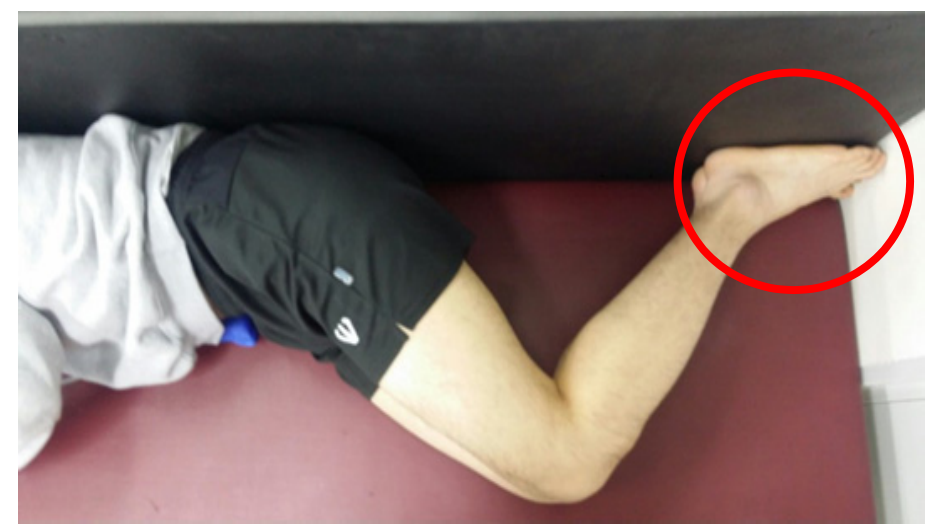

Fig. 2. Modified clamshell exercise (CLAM) 1; the back is in contact with the wall and the feet are pushed at about $10 \%$ of the maximal contraction against the wall

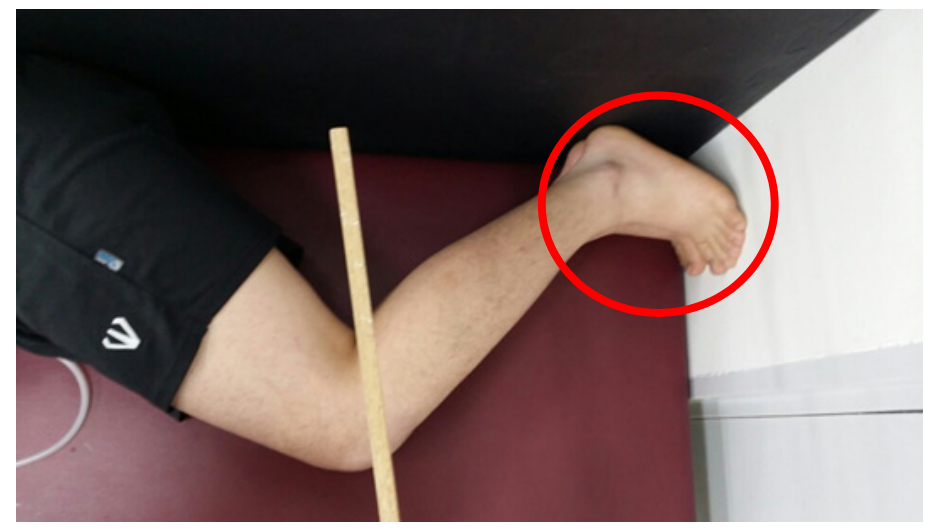

Fig. 3. Modified clamshell exercise (CLAM) 2, both heels and metatarsal heads are pushed at about $10 \%$ of the maximal contraction against the wall, while maintaining neutral ankle position

changes in the body's position during the exercise, and has been reported to decrease substitution from the QL and to increase the GMED activity during the side-lying abduction exercise [4]. The stabilizer pressure bio-feedback unit was inflated until the pressure reached $40 \mathrm{mmHg}$, after which the participant and the investigator (JSG) observed this pressure to ensure that it maintained between 35 and $45 \mathrm{mmHg}$ during the exercises.

\section{2) Modified CLAM 1}

To conduct the modified CLAM 1, participants assumed the same position as the standard CLAM, but their back touched the wall and they pushed their feet at about $10 \%$ of the maximal contraction against the wall (Fig. 2), separated their knees, and rotated the weak limb upward keeping their feet together.

\section{3) Modified CLAM 2}

To perform the modified CLAM 2, the participants remained in the modified CLAM 1 position and pushed both the heels and metatarsal heads against the wall at about $10 \%$ of the maximal contraction while maintaining neutral ankle position (Fig. 3), separated their knees, and rotated the weak limb upward keeping their feet together. 

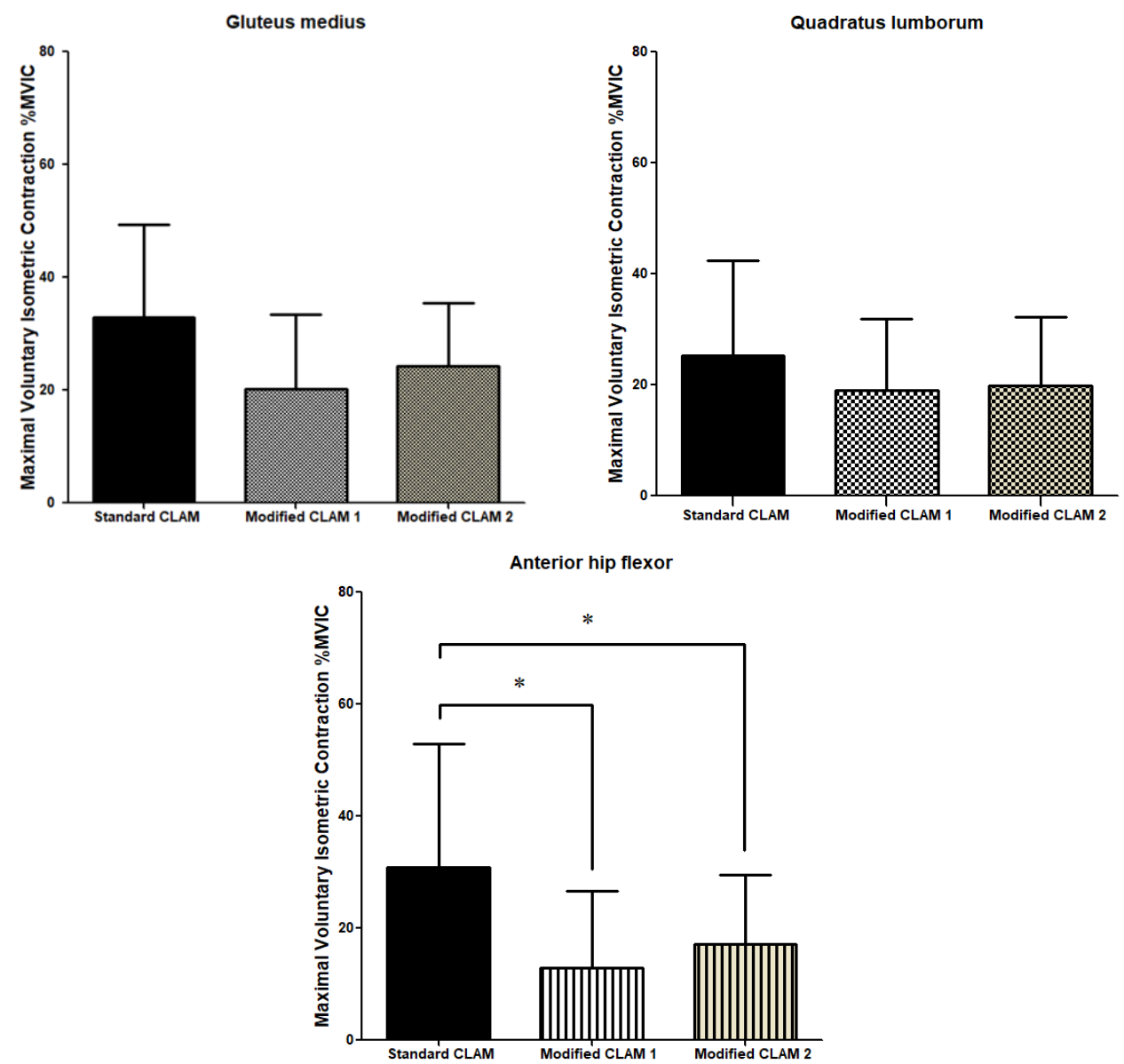

Fig. 4. Comparison of the GMED, QL, and AHF muscle activities between the three exercises (standard and modified clamshell exercises 1 and 2)

Abbreviations: \% MVIC, percentage of maximal voluntary isometric contractions; GMED, gluteus medius; QL, quadratus lumborum; AHF, anterior hip flexor. ${ }^{*} p<.017$

\section{Statistical analysis}

The Statistical Package for the Social Sciences for Windows version 18.0 (SPSS, Chicago, IL, USA) was used for all statistical analyses. One-way, repeated-measures analysis of variance was used to assess the statistical signifi cance of the GMED, QL, and AHF EMG activities, as well as the GMED/AHF and GMED/QL EMG activity ratios during the CLAM with different foot positions. The level of significance was set at .05 . If a significant difference was found, a Bonferroni adjustment (or correction) was performed (with $\mathrm{a}=.05 / 3=.017$ ).

\section{Results}

1. GMED, QL, and AHF muscle activity

Fig. 4 shows the \% MVIC of the GMED, QL, and AHF during the three types of CLAM exercises. Neither the GMED nor the QL muscle activities differed significantly between the standard CLAM and the modified CLAM for the two different foot positions ( $\mathrm{F}=4.74, \mathrm{P}=.02$; $\mathrm{F}=4.57, \mathrm{P}=.02$, respectively). However, there were significant differences between the AHF activities during the three types of CLAM exercises $(\mathrm{F}=11.17, \mathrm{P}=.00)$. 

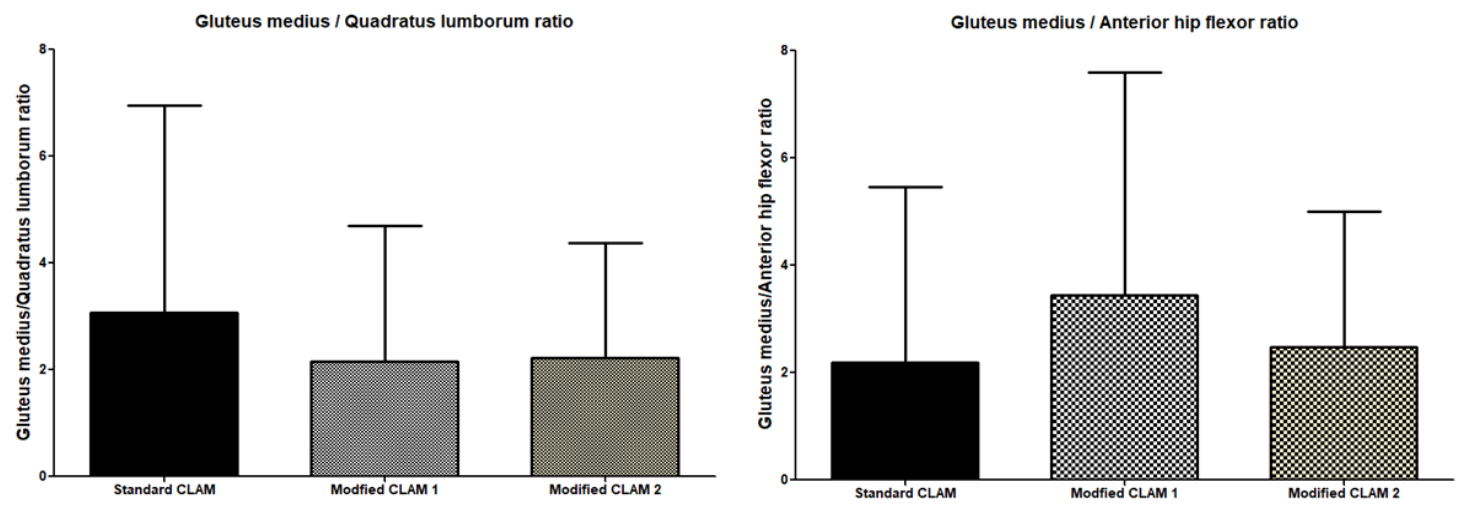

Fig. 5. Comparison of the gluteus medius/quadratus lumborum and gluteus medius/anterior hip flexor ratios between the 3 exercises (standard, and modified clamshell exercises 1 and 2). ${ }^{*} p<.017$

Modified CLAM 1 and 2 showed significantly less AHF activity than that during the standard CLAM $(\mathrm{P}=.00, \mathrm{P}=.01$, respectively); whereas, there was no significant difference between AHF activities during the modified CLAM with the two different foot positions $(\mathrm{P}=.09)$.

\section{GMED/QL and GMED/AHF ratios}

The GMED/QL and GMED/AHF ratios during the three types of CLAM exercises are shown in Fig. 5. The GMED/QL and GMED/AHF ratios were not significantly different among all types of CLAM ( $\mathrm{F}=.63, \mathrm{P}=.55 ; \mathrm{F}=.82$, $\mathrm{P}=.45$, respectively).

\section{Discussion}

This study was conducted to investigate whether the GMED, QL, and AHF activities and the GMED/QL and GMED/AHF muscle activity ratios differed between the standard CLAM and 2 modified CLAM exercises performed by participants with GMED weakness. The GMED and QL activities and the GMED/QL and GMED/AHF activity ratios were not significantly different among the three types of CLAM. However, the AHF activity was significantly lower during the two modified CLAM exercises than in the standard CLAM. Nevertheless, to our knowledge, this is the first study to compare the GMED, QL, and AHF activities and the GMED/QL and GMED/AHF activity ratios during different CLAM exercises in participants with GMED weakness. The results of the current study partially supported the research hypotheses.

Contrary to the research hypothesis, the GMED activity did not increase during the two modified CLAM exercises when compared to the standard CLAM. This result is similar with respect to the findings of a previous study that showed the standard CLAM does not produce high activation of the GMED and activates the TFL and AHF to a greater extent, and that the AHF is more active than the TFL and GMED [15]. In addition, in this study, two different foot positions in the modified CLAM were designed to reduce the AHF activity without enhancing the GMED activity. Although gluteus maximus activity was not investigated in this study, it is possible that the gluteus maximus activity would have been increased during the modified CLAM exercises via reciprocal inhibition.

The QL activity also did not decrease during the two modified CLAM exercises when compared to the standard CLAM. The PI postulated that pushing the feet against the wall during the modified CLAM exercises would inhibit pelvic elevation. However, the results of this study did 
not validate the research hypothesis. Specifically, plantar flexion did not contribute to activation of the QL. Moreover, in this study, all three CLAM exercises included the stabilizer pressure biofeedback unit to minimize the QL muscle activity and pelvic rotation during the exercises $[4,11]$. This lumbopelvic stabilization would have been sufficient to stabilize hip hiking, which can be caused by an overactive QL during CLAM exercises.

The AHF activity was significantly lower during the two modified CLAM exercises than in the standard CLAM (by $38.41 \%$ and $26.49 \%$ for CLAM 1 and 2, respectively). These results support our research hypothesis, and may be explained by the mechanism of reciprocal inhibition. Reciprocal inhibition occurs because of afferent inputs from the agonist muscle spindles stimulating an inhibitory interneuron in the spinal cord, which causes inhibition of the alpha motor neuron of the antagonist muscle $[16,17]$. Isometric plantar flexion contraction during the modified CLAM may have activated posterior chain muscles in the lower extremities and reciprocally inhibited the antagonist muscles (i.e., AHF). These results suggest that AHF muscle activity can be decreased by reciprocal inhibition $[16,17]$. However, the ankle joint position (between the modified CLAM 1 and 2) did not influence the AHF activity. In the present study, the foot position was standardized in two different ways because a different ankle angle could affect the lower limb muscle activity, and the PI thought that the subjects would feel comfortable and would be able to push their feet against wall in a neutral ankle position.

It should be noted that this study had several limitations. First, the age range of the participants was limited to 18-24 years; therefore, our outcomes may not be generalized to the population belonging to other age groups. Second, this study is a cross-sectional study; therefore, the long-term effects of different foot conditions on the muscles that were investigated cannot be determined. Third, the upward movement of the pelvis during the exercises was not well controlled. Fourth, the strength of the push at about 10\% of the maximal contraction was not measured. Further studies should determine the long-term effects on the GMED and QL muscle activities in different foot positions in subjects with GMED weakness.

\section{Conclusion}

This study examined the effects of the modified CLAM on the EMG amplitude of the GMED, QL, AHF activities, as well as the GMED/QL and GMED/AHF ratios in the participants with weak GMED. Our results showed that the AHF muscle activity significantly decreased during the modified CLAM when compared to that during the standard CLAM. Therefore, this finding signifies that the modified CLAM can be recommended as a good method to minimize the AHF activity while maintaining the GMED activity in subjects with weak GMED.

\section{References}

[1] Lee D. Instability of the sacroiliac joint and the consequences to gait. J Man Manip Ther. 1996;4(1):22-29.

[2] Gottschalk F, Kourosh S, Leveau B. The functional anatomy of tensor fasciae latae and gluteus medius and minimus. J Anat. 1989;166:179.

[3] Bolgla LA, Uhl TL. Electromyographic analysis of hip rehabilitation exercises in a group of healthy subjects. J Orthop Sports Phys Ther. 2005;35(8):487-94.

[4] Cynn HS, Oh JS, Kwon OY, et al. Effects of lumbar stabilization using a pressure biofeedback unit on muscle activity and lateral pelvic tilt during hip abduction in sidelying. Arch Phys Med Rehabil. 2006;87(11):1454-8.

[5] Janda V, Frank C, Liebenson C. Evaluation of muscular imbalance. Rehabilitation of the spine: A practitioner's manual. 1996;6:97-112.

[6] Powers CM. The influence of abnormal hip mechanics on knee injury: A biomechanical perspective. J Orthop Sports Phys Ther. 2010;40(2):42-51. 
[7] Chaitow L, Crenshaw K. Muscle energy techniques. Elsevier Health Sciences, 1996.

[8] Sahrmann S. Diagnosis and treatment of movement impairment syndromes. St.Louis, Msby. 2002:193-247.

[9] Shamberger W. The Malalignment Syndrome: Implications for medicine and sport. Churchill Livingstone. 2002.

[10] Richardson C, Jull, Gwendolen, et al. Therapeutic exercise for spinal segmental stabilization in low back pain. London, Churchill Livingstone. 1999:211-22.

[11] Park KM, Kim SY, Oh DW. Effects of the pelvic compression belt on gluteus medius, quadratus lumborum, and lumbar multifidus activities during side-lying hip abduction. J Electromyography Kinesiol. 2010;20(6): 1141-5.

[12] McGill, Stuart. Low Back Disorders: Evidence-based prevention and rehabilitation. Human Kinetics. 2007.

[13] Natalie Sidorkewicz, Edward D.J. Cambridge, Stuart M. McGill. Examining the effects of altering hip orientation on gluteus medius and tensor fasciae latae interplay during common non-weight-bearing hip rehabilitation exercises. Clinical Biomechanics, 2014;29(9):971-6.

[14] Selkowitz DM, Beneck GJ, Powers CM. Which exercises target the gluteal muscles while minimizing activation of the tensor fascia lata? Electromyographic assessment using fine-wire electrodes. J. Orthop. Sports Phys. Ther. 2013;43(2):54-64.

[15] Joseph M McBeth, Jennifer E. Earl-Boehm, Stephen C. Cobb, et al. Hip Muscle Activity During 3 Side-Lying Hip Strengthening Exercises in Distance Runners. Journal of Athletic Training 2012:47(1):15-23.

[16] Leonard CT. Principles of reflex action and motor control. 1st ed. St. Louis, Mosby, 1997:70-99.

[17] Stuart I. Human physiology: mechanism of contraction and neural control. 10th ed. New York, McGraw-Hill. 2007.

[18] Kim SJ, Kwon OY, Yi CH, et al. Comparison of abdominal muscle activity during a single-legged hold in the hook-lying position on the floor and on a round foam roll. National Athletic Trainers' Association, Inc. 2011;46:403-8.

[19] Khayambashi K, Mohammadkhani Z, Ghaznavi K, et al. The effects of isolated hip abductor and external rotator muscle strengthening on pain, health status, and hip strength in females with patellofemoral pain: A randomized controlled trial. J Orthop Sports Phys Ther. 2012;42(1):22-9.

[20] Fredericson M, Cookingham CL, Chaudhari AM, et al. Hip abductor weakness in distance runners with iliotibial band syndrome. Clin J Sport Med. 2000;10(3):169-75.

[21] Friel K, McLean N, Myers C, Caceres M. Ipsilateral hip abductor weakness after inversion ankle sprain. J Athl Train. 2006;41(1):74.

[22] Kendall F, McCreary E, Provance P, et al. Muscles testing and function with posture and pain. Philadelphia; Lippincott Williams \& Wilkins. 2005.

[23] Bewyer KJ, Bewyer DC, Messenger D, et al. Pilot data: Association between gluteus medius weakness and low back pain during pregnancy. Iowa Orthop J. 2009;29:97.

[24] Wong YM. Skin thickness and VMO:VL ratio. J Orthop Sports Phys Ther 1999;29:686.

[25] Flegal KM, Carroll MD, Kuczmarski RJ, et al. Overweight and obesity in the United States: Prevalence and trends, 1960-1994. IJO. 1998;22(1):39.

[26] Cram J, Kasman G. Introduction to Surface Electromyography. Aspen, Gaithersburg. 1998.

[27] Hermens HJ, Freriks B, Disselhorst-Klug C, et al. Development of recommendations for SEMG sensors and sensor placement procedures. J Electromyography Kinesiol. 2000;10(5):361-74.

[28] Ayotte NW, Stetts DM, Keenan G, et al. Electromyographical analysis of selected lower extremity muscles during 5 unilateral weight-bearing exercises. J Orthop Sports Phys Ther. 2007;37(2):48-55.

[29] Soderberg GL, Knutson LM. A guide for use and interpretation of kinesiologic electromyographic data. Physical therapy. 2000;80(5):485-98. 
[30] Nyland J, Kuzemchek S, Parks M, et al. Femoral anteversion influences vastus medialis and gluteus medius EMG amplitude: Composite hip abductor EMG amplitude ratios during isometric combined hip abductionexternal rotation. J Electromyography Kinesiol. 2004; 14(2):255-61.
[31] Sykes K, Wong YM. Electrical activity of vastus medialis oblique muscle in straight leg raise exercise with different angles of hip rotation. Physiotherapy. 2003;89(7):423-30.

[32] De Luca CJ. The use of surface electromyography in biomechanics. J Appl Biomech. 1997;13(2):135-63. 